\title{
Kinematic Evaluation of 4 Pediatric Collars and Distribution of Cervical Movement Between Primary and Coupled Angles
}

\author{
Ayman Assi, PhD, ${ }^{*}+\dagger$ Paul Yazbeck, MD, $\S$ Abir Massaad, PT, MSc, $\dagger$ \\ Wafa Skalli, PhD, $\ddagger$ and Ismat Ghanem, MD, MSc* $†$
}

\begin{abstract}
Background: Primary and coupled angle restrictions, when neck collars are used, have been investigated mainly in adults and not yet in children.

Purpose: To evaluate the efficiency of 4 pediatric collars in reducing cervical range of motion (ROM) in primary and coupled planes.
\end{abstract}

Methods: Thirty asymptomatic children (16 boys and 14 girls) aged 6 to 12 years participated in the study. A motion analysis system was used to evaluate the ROM of the cervical spine during flexion/extension, left and right lateral bending, and left and right axial rotation. Primary and coupled ROM were evaluated in unbraced and braced conditions. Four cervical collars were tested: Philadelphia, Miami Jr, Necloc, and the conventional Hard Collar. Thirteen subjects were tested 2 times to evaluate the repeatability of the parameters. The ROM in each plane was normalized to the sum of the ROM in the 3 planes, for each movement, to estimate the percentage of the movement in each plane (normalized ROM), in braced and unbraced conditions. The analysis of variance and post hoc Benferroni tests were applied on raw and normalized ROM.

Results: ROM collected in collars showed a significant difference compared with the unbraced condition. ROM obtained in Necloc and Miami Jr showed a significant difference compared with Philadelphia and conventional Hard Collar. The primary plane is activated at $80 \%$ during flexion-extension and left-right axial rotation; however, $55 \%$ of the total movement was completed in the frontal plane during left-right lateral bending in unbraced condition. Statistical differences in the normalized ROM were found between the braced and unbraced conditions and among collars.

Conclusions: Necloc and Miami Jr presented the highest limitation of movement in the primary and secondary planes. The distribution strategy of a movement, between primary and

From the *Laboratory of Biomechanics, School of Medicine, PTS Campus of Innovation and Sport; $†$ Gait and Motion Analysis Laboratory, SESOBEL; §Hôtel Dieu de France Hospital, Beirut, Lebanon; and \$Laboratoire de Biomécanique, Arts et Métiers ParisTech, Paris, France.

The authors declare no conflicts of interest.

Reprints: Ayman Assi, PhD, Laboratory of Biomechanics, School of Medicine, PTS Campus of Innovation and Sport, Damascus Street, BP 17-5208 Mar Mikhael, Beirut, Lebanon. E-mail: ayman.assi@ usi.edu.lb. coupled angles, is different between the braced and unbraced conditions.

Key Words: biomechanics, 3D motion analysis, cervical spine, children, coupled movement, collars

C ervical collars are usually prescribed for correction intervertebral segment stabilization, treatment of posttraumatic injuries, and postoperative immobilization. ${ }^{1-3}$ Several cervical collars are used in adults with varying degrees of immobilization of neck movement. These orthoses are widely described in adults ${ }^{4-9}$ based on kinematic evaluation. However, there are no studies that have analyzed the extent of immobilization achieved by cervical collars in children younger than 15 years of age.

To evaluate the immobilization of the cervical spine, it is essential to explore normal cervical range of motion (ROM). Most of the studies reported normal cervical ROM in the adult population ${ }^{10-16}$ and only a few studies, where video graphs and inclinometers were used to assess active cervical $\operatorname{ROM}^{17,18}$ or passive kinematics, ${ }^{19}$ had reported normal data on children. The cervical spine, especially the upper part, represents a unique anatomy compared with other regions of the spine and displays a combination of motions, known as coupled motions. ${ }^{20}$ When a movement is performed primarily in a plane (ie, flexion-extension in the sagittal plane), the movements described in the other planes (ie, frontal and horizontal planes) are called coupled angles/movements. Coupled motions are mainly reported in adults and not well described in children. ${ }^{15,16,21,22}$ Moreover, restriction of coupled motions by cervical collars has never been investigated.

The aim of this study was to evaluate the restriction of primary and coupled neck movements provided by 4 different types of cervical collars prescribed for children.

\section{METHODS}

\section{Subjects}

Thirty healthy children, 16 boys and 14 girls, aged between 6 and 12 years [mean (SD): 9.3 (2)] participated 
in the study. Anthropometric measurements were collected: weight [37 kg (12)], height [138 $\mathrm{cm}$ (12.4)], neck circumference [26.6 $\mathrm{cm}(3.5)]$, and shoulder girdle width [27.6 cm (2.8)]. Children with previous history of pain, trauma, or orthopaedic disorder were excluded. The subjects were recruited from the SOS Children's Village association in Lebanon. Parents and the association's manager signed the consent form. The Institutional Review Board approved the study design.

\section{Equipment and Protocol}

Motion acquisitions were performed in a Gait laboratory equipped with 6 infrared MX3 cameras with a frequency set at $200 \mathrm{~Hz}$ (Vicon Motion Systems, Oxford, UK). The system was calibrated before each acquisition (mean residual error on 3D marker reconstruction: $0.8 \mathrm{~mm}$ ). The marker set of the Plug in Gait protocol was used for the upper limbs, including head and thorax, based on the Davis protocol ${ }^{23}$ : 4 markers were placed on the head (left and right front head and back head), spinous process of the seventh cervical vertebrae, spinous process of the tenth thoracic vertebrae, jugular notch where the clavicles meet the sternum, xiphoid process of the sternum, and 1 marker on the middle of the right scapula (to create an asymmetry that helps the autolabeling routine). Seven markers were placed bilaterally: acromioclavicular joint, upper arm (between the elbow and the shoulder marker), lateral epicondyle approximating elbow joint axis, lower arm (between the wrist and the elbow marker), wrist thumb side, most caudallateral point on the radial styloid, most caudal-medial point on the ulnar styloid, and finger (placed on the dorsum of the hand just below the head of the second metacarpal) (Fig. 1A). Eight segments were reconstructed: head, thorax, arm, forearm, hand bilaterally. Markers were placed by the same physiotherapist.

\section{Collars' Evaluation}

The 4 most used cervical collars in our pediatric service were tested: Philadelphia, Miami Jr, Necloc, and the conventional Hard Collar (Fig. 1B). An average size of collars was chosen for all subjects as neck circumference had a small intervariability. Collars were placed, by the same physiotherapist who placed the reflective markers, whereas subjects were in a standing position. The first acquisition was performed without a collar, referred to in the text as the unbraced condition. Then, the same acquisitions were performed while the subjects were wearing the collars. The order of testing of the collars was randomized.

\section{Experimental Protocol}

Motion analysis measurements were taken in an illuminated closed room without sun light. Subjects were bare torso (some of the female subjects wore the upper part of a 2-piece swimsuit) and were sat in an ordinary school chair, feet flat on the floor with a back support without inclination. ${ }^{13}$ Their knees were bent at 90 degrees and their hands were resting on their thighs. The starting position was the neutral position, defined by the subject. A static trial was performed, where the subject was asked not to move for 5 seconds. Then, the subject was asked to perform single-plane neck movements in the following order: flexion, extension, left lateral bending, right lateral bending, left axial rotation, and right axial rotation (Fig. 2). The neutral position was held for 3 seconds between each movement. ${ }^{8}$ The reproducibility of the neutral position was not evaluated. The subjects were asked to perform neck motion without moving their chest and their shoulders by bringing their chin to their sternum, then, their occiput to their back during flexion-extension, their ear to their shoulder during lateral bending (bilaterally), and turning their nose during axial rotation (bilaterally). During the test, subjects were asked for maximal effort to obtain maximal-end cervical ROM and good reliability of measurement in accordance with the recommended procedures. ${ }^{8,10}$ Familiarization trials were performed before acquisitions. To study the repeatability of the protocol, 13 subjects randomly chosen within this sample performed the exam twice. Sessions were separated by at least 1 week.

\section{ROM Calculation}

The movement of the neck was calculated as the movement of the head relatively to the thorax. Raw data were processed using the pipeline in Workstation (Vicon Motion Systems): fill gap routine (at 20 frames) and the Woltring filter with a scale of 10 . Three-dimensional neck angles were calculated using the YXZ sequence of Cardan between the head and the thorax positions and extracted from each trial in an Excel Sheet. The movements were regrouped in 3 graphs where curves were sampled into 51 points and defined as following: total flexion-extension (TFE), left and right lateral bending noted as total lateral bending (TLB), left and right axial rotation noted as total axial rotation (TAR). The cervical ROM was calculated on each curve. Primary and coupled movements were explored in the plane of interest and the 2 remaining planes for each neck motion. Percentages of motion reduction in primary and secondary planes were calculated for each collar among the population.

To investigate the coupled angles during a singleplane movement in both braced and unbraced conditions, all ROM were normalized to the sum of ROM that the subject performed in all planes during a task, that is, during flexion-extension of the neck, the angles described in the 3 planes were S_TFE, F_TFE, and H_TFE (for the sagittal, frontal, and horizontal planes, respectively), their sum is noted Total_TFE and the normalized ROM will be: $\% \mathrm{~S} \_$TFE $=\mathrm{S} \_$TFE$/$Total_TFE, $\% \mathrm{~F}$-TFE $=\mathrm{F} \_$TFE $/$ Total_TFE, and $\%$ H_TFE $=$ H_TFE/Total_TFE. Mean and $\overline{\mathrm{SD}}$ of percentages were calculated for the 30 asymptomatic children (Fig. 3).

\section{Statistics}

The normality of the distribution of values was tested using the Shapiro-Wilk test. Mean values and SD of cervical ROM were calculated for the 30 healthy subjects in the unbraced condition and when collars were used (Table 1). Test-retest repeatability was evaluated for 
A
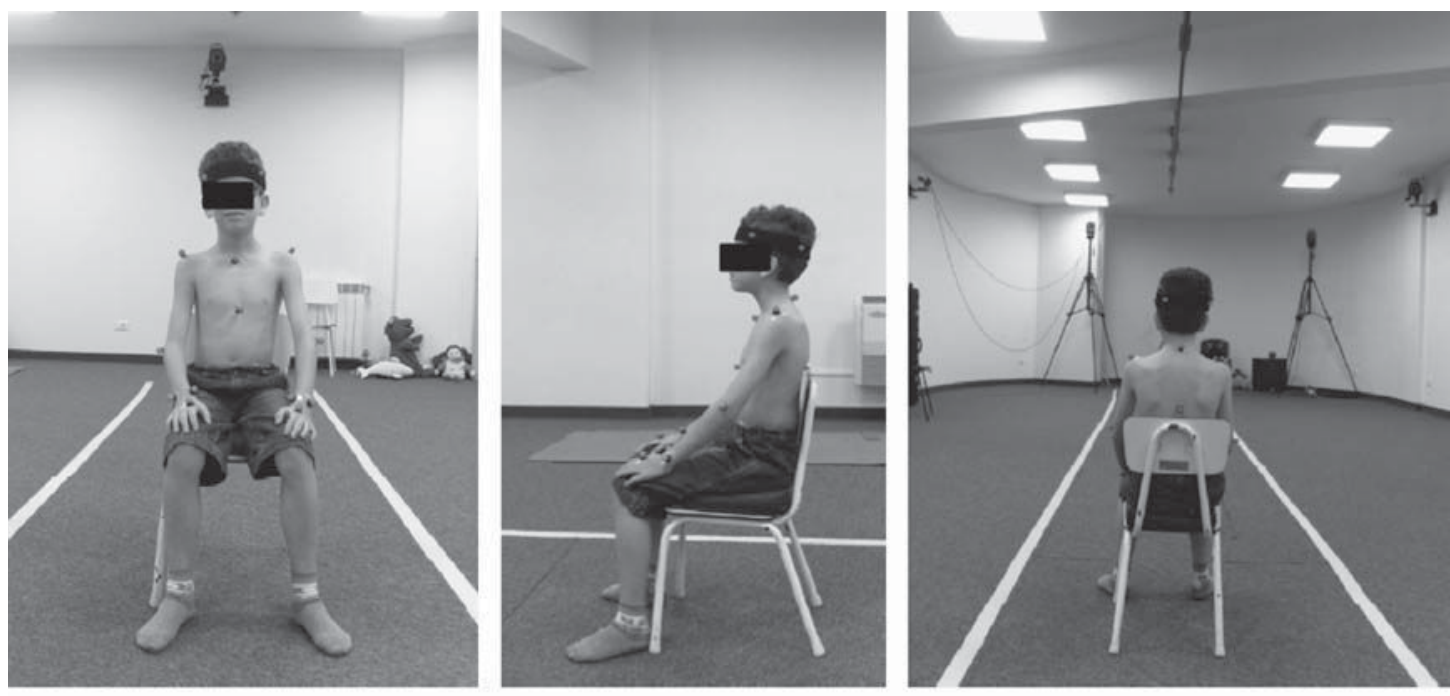

B

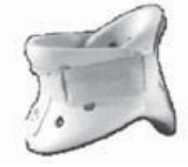

Philadelphia

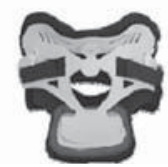

Miami Jr.

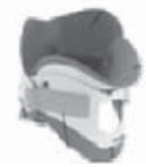

Necloc

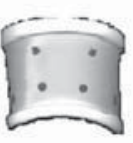

Hard Collar

FIGURE 1. A, The testing set up and the markers' placement based on the Davis protocol. B, The 4 pediatric collars: Philadelphia, Miami Jr, Necloc, and Hard Collar.

each ROM, in each plane and for each movement by calculating the intraclass correlation coefficient (ICC) between the 2 sessions. The confidence interval at $95 \%$ (CI 95\%) was evaluated for each ROM. ${ }^{24}$ The $t$ test of Student was applied to estimate significant differences between sessions (level $P<0.05$ ). ROM measurements and percentages of ROM were compared with a 1-way analysis of variance. A Bonferroni correction was applied for multiple comparisons, setting significance at $P \leq 0.005$. Data processing was performed in MATLAB (Mathworks, Natick, MA). Statistics were calculated using XLSTAT (Addinsoft, New York, NY).

\section{RESULTS}

\section{Database for Normal Kinematics}

Cervical ROM in primary and secondary planes of interest during TFE of the neck, TLB, and TAR has been reported in Table 1 (in unbraced condition and when collars were used). The maximal contribution of coupled movements was found during TLB: with a mean ROM of 84 degrees in the frontal plane, and coupled motion of 29 and 43 degrees, respectively, in sagittal and horizontal plane.

\section{Repeatability}

The analysis of intersession variability revealed that there were no significant differences, between sessions and for each movement: $P$-value ranged between 0.1 and 0.9 for all planes of interest during the performed movements. The ICC ranged between 0.6 and 1 except for the ROM in the sagittal plane, during TLB, and in the unbraced condition, where the ICC was around 0.55 . The CI at $95 \%$ was \pm 10 to \pm 11 degrees for the primary angles in TFE and TLB and \pm 14 degrees for TAR for unbraced and braced conditions. CIs for coupled ROM ranged between \pm 4 and \pm 7 degrees (the larger CIs were found for TLB in the horizontal plane and TAR in the frontal plane).

\section{Cervical ROM in Braced Conditions}

The ROM obtained during neck flexion-extension, lateral bending, and axial rotation for each collar was collected by plane of motion in Table 1. All ROM performed in the collar-wearing condition were significantly different to the unbraced condition. The comparison of ROM between collars highlighted significant differences (Table 1).

During flexion-extension, the conventional Hard Collar had a significantly larger ROM in the sagittal plane than the Necloc $(P<0.0001)$. No statistical differences between collars were found for ROM in the secondary planes, during flexion-extension.

During left and right lateral bending, Philadelphia had a significantly larger ROM in the frontal plane than any of the other collars $(P<0.0001)$. Coupled movements occurring in the horizontal plane for Miami $\mathrm{Jr}$ and the Hard Collar were significantly smaller $(P=0.001$ and 

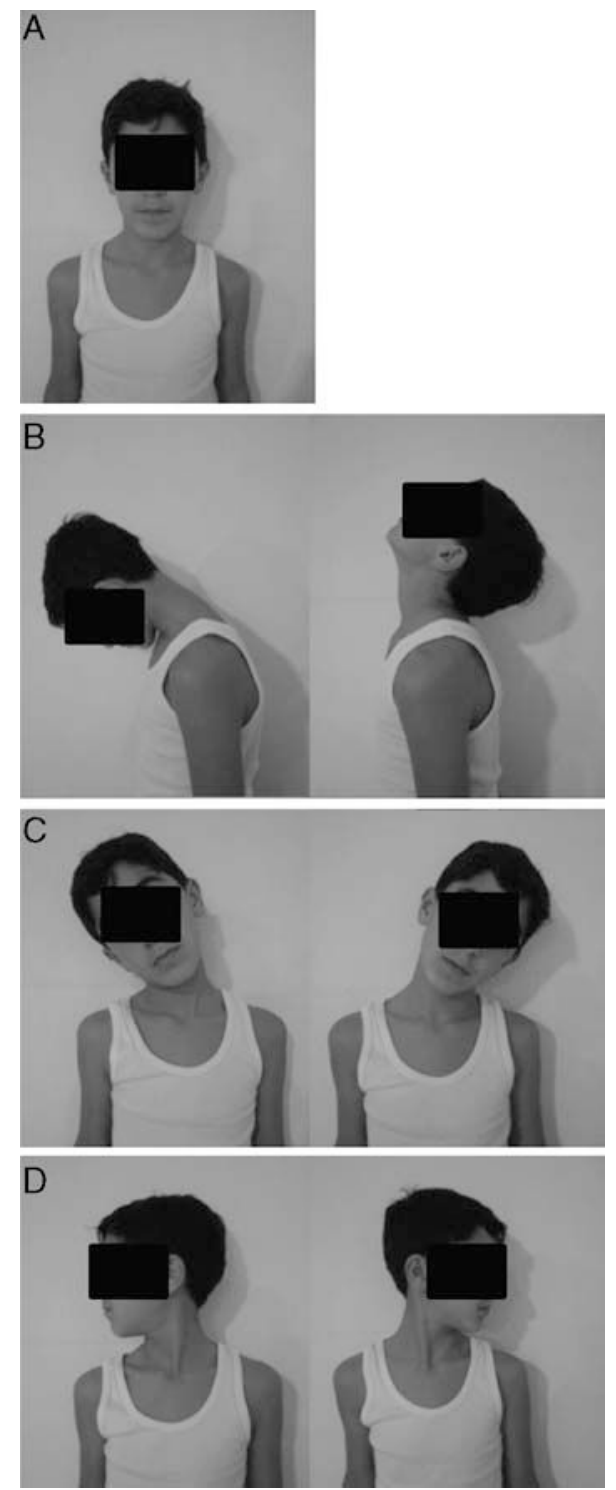

FIGURE 2. Neck movements: (A) static, (B) flexion/extension, (C) left/right lateral bending, and (D) left/right axial rotation.

$P=0.003$, respectively) to Philadelphia. There was no significant difference between Necloc, Miami Jr, and the Hard Collar, in primary and secondary planes, during left and right lateral bending.

During left and right axial rotation of the neck, Miami Jr and Necloc had significantly lower ROM in the horizontal plane than Philadelphia and the Hard Collar $(P<0.004)$. No differences were found in the secondary planes, during left and right axial rotation.

Similar results of statistical differences between collars were found when multiple comparisons were applied on the percentage of motion reduction.

\section{Normalized Primary and Coupled Angles}

Percentages of ROM, when normalized to the total movement performed in the 3 planes, were collected in Figure 3. The analysis of the normalized ROM, between the braced and unbraced conditions, revealed significant differences in the distribution strategy of primary and coupled angles.

During flexion-extension, the movement was performed at $>80 \%$ in the sagittal plane (primary plane) in the unbraced condition, whereas the rest of the movement was completed at $<10 \%$ in the frontal and horizontal planes. The proportion of sagittal and frontal movements were significantly lower in all the braced conditions compared with the unbraced condition $(P<0.004)$, during TFE. Necloc and Hard Collar showed a significantly lower $\%$ of movement performed in the horizontal plane during TFE, when compared with the unbraced condition $(P<0.004)$. However, no significant differences were found in the normalized ROM in the horizontal plane during TFE of the neck between Philadelphia, Miami Jr, and the unbraced condition.

During left and right lateral bending, $55 \%$ of the movement was performed in the frontal plane (primary plane) in the unbraced condition and $18 \%$ and $26 \%$ were performed in the secondary planes (for the sagittal and horizontal planes, respectively). No statistical differences were found in the normalized ROM, in each plane, between Philadelphia, Necloc, and the unbraced condition in the 3 planes. The normalized ROM in the frontal plane was significantly more important with Miami Jr and Hard Collar, when compared with the unbraced condition and Necloc $(P<0.001)$. The same results were found in the horizontal plane, in addition to a significant difference with Philadelphia $(P<0.003)$.

During axial rotation, in the horizontal plane (primary plane), the movement was performed at $78 \%$ in the unbraced condition, whereas in the secondary planes the movement was performed at $9 \%$ and $13 \%$ (for the sagittal and frontal planes, respectively). All collars showed significantly lower normalized ROM in the horizontal plane and significantly larger normalized ROM in the sagittal and frontal planes compared with the unbraced condition $(P<0.0001)$. Philadelphia and the Hard Collar showed significantly lower normalized ROM in the frontal plane compared with Necloc and Miami Jr. Both Philadelphia and the Hard Collar showed significantly larger normalized ROM than Necloc, in the horizontal plane, whereas only Philadelphia showed a larger normalized ROM than Miami Jr.

\section{DISCUSSION}

Restriction of both primary and coupled movements of the cervical spine is not well described in children. The purpose of this study was to compare the efficiency of 4 pediatric cervical collars (Philadelphia, Miami Jr, Necloc, and the conventional Hard Collar) in the restriction of primary and coupled movements of the neck using a motion analysis system. Thirty asymptomatic children, aged between 6 and 12 years, performed neck flexion-extension, left-right lateral bending, and 


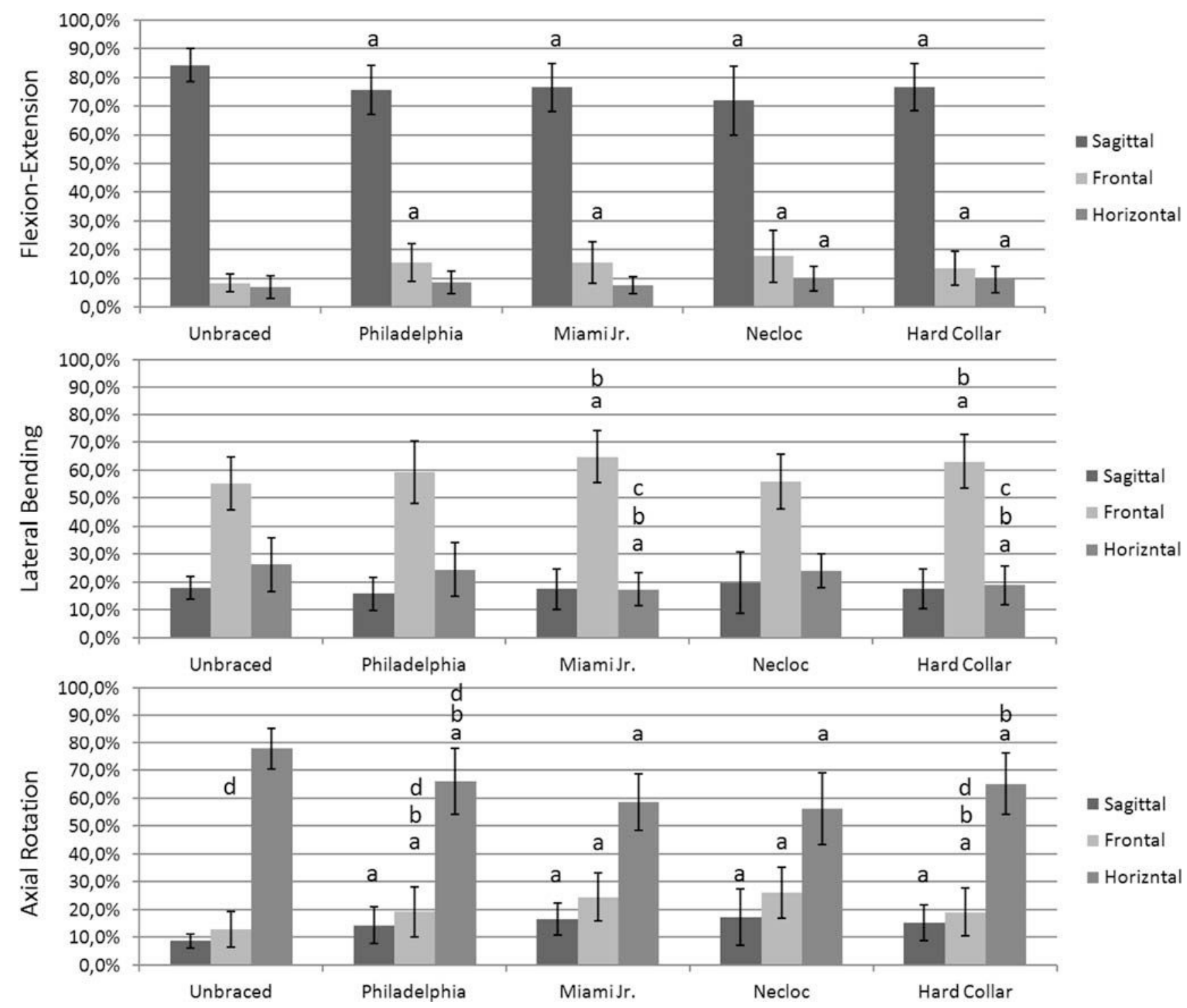

FIGURE 3. Normalized range of motion in the unbraced and braced conditions (Philadelphia, Miami Jr, Necloc, and the Hard Collar), tested by 30 asymptomatic children aged 6 to 12 years during flexion-extension, left and right lateral bending as well as left and right axial rotation in the frontal, sagittal, and horizontal planes. Significant differences: a, with unbraced condition; b, with Necloc; c, with Philadelphia; d, with Miami Jr.

left-right axial rotation in the unbraced and braced conditions, where the 4 collars were tested.

Most of the studies have reported normal cervical ROM in the adult population ${ }^{10-16}$ and only a few studies have reported normal data in children. ${ }^{17,18}$ The values of primary ROM found in our experiment were comparable with these previous studies on children.

Many authors have emphasized the importance of the effect of sex and age on cervical ROM, in the adult population. ${ }^{19,22,25}$ These effects were not evaluated in our study because of the small sample size.

Coupled movements were mainly described in adults. $15,16,21,26,27$ This is the first study where coupled movements were described in children aged between 6 and
12 years. Our results seem to be different to those measured in 15- and 16-year-old subjects ${ }^{16}$ : around 5 degrees of difference in the coupled movements and 15 degrees in the primary movements. This difference could be related to the variety of ages in our population or to a different performance of the movement in the young subjects included in our study.

In this study, a test-retest assessment was conducted on 13 subjects. The kinematic parameters were shown to be repeatable. The low repeatability of ROM in the sagittal plane during left-right lateral bending in the unbraced condition could be related to incapacity of the children to reproduce the same movement which led them to use a different strategy of distribution of primary and 
TABLE 1. Average Cervical ROM and SD on 30 Healthy Children Aged 6 to 12 Years During Flexion-Extension, Left and Right Lateral Bending, and Left and Right Axial Rotation in the 3 Planes

\begin{tabular}{|c|c|c|c|}
\hline $\begin{array}{l}\text { Planes } \\
\text { Movements }\end{array}$ & $\begin{array}{l}\text { Sagittal (deg.) } \\
\text { Mean (SD) } \\
\end{array}$ & $\begin{array}{l}\text { Frontal (deg.) } \\
\text { Mean (SD) }\end{array}$ & $\begin{array}{c}\text { Horizontal (deg.) } \\
\text { Mean (SD) } \\
\end{array}$ \\
\hline \multicolumn{4}{|c|}{ Flexion-extension } \\
\hline Unbraced & $118(20)$ & $12(7)$ & $11(8)$ \\
\hline Philadelphia & $32(13)^{*}$ & $6(3) *$ & $3(1)^{*}$ \\
\hline Miami Jr & $32(15) *$ & $6(4)^{*}$ & $3(2)^{*}$ \\
\hline Necloc & $27(13)^{*}$ & $7(5) *$ & $4(2)^{*}$ \\
\hline Hard Collar & $39(18)^{* \dagger}$ & $7(5)^{*}$ & $6(4)^{*}$ \\
\hline \multicolumn{4}{|c|}{ Left-right lateral bending } \\
\hline Unbraced & $29(10)$ & $84(14)$ & $43(22)$ \\
\hline Philadelphia & $12(8)^{*}$ & $44(18)^{*}$ & $18(10)^{*}$ \\
\hline Miami Jr & $8(4) *$ & $33(15) * t$ & $9(5) *$ \\
\hline Necloc & $11(7)^{*}$ & $31(14) *$ & $14(8) *$ \\
\hline Hard Collar & $9(4)^{*}$ & $32(14)^{*}+$ & $10(6)^{*} t$ \\
\hline \multicolumn{4}{|c|}{ Left-right axial rotation } \\
\hline Unbraced & $15(6)$ & $23(14)$ & $134(17)$ \\
\hline Philadelphia & $9(4)^{*}$ & $14(9)^{*}$ & $47(25)^{*}$ \\
\hline Miami Jr & $8(4)^{*}$ & $12(8)^{*}$ & $28(13)^{*} \ddagger$ \\
\hline Necloc & $8(6) *$ & $13(9)^{*}$ & $28(16)^{*}+$ \\
\hline Hard Collar & $10(5)^{*}$ & $12(7)^{*}$ & $43(20)^{*} \dagger \S$ \\
\hline \multicolumn{4}{|c|}{$\begin{array}{l}\text { Unbraced and braced conditions: evaluation of } 4 \text { collars. } \\
\text { *Significantly different from unbraced. } \\
\text { †Significantly different from Necloc. } \\
\text { †Significantly different from Philadelphia. } \\
\text { §Significantly different from Miami Jr. }\end{array}$} \\
\hline
\end{tabular}

coupled angles, during the performance of the movement. The reproducibility of the neutral position between tasks should be evaluated for a better understanding of proprioception behaviors in the pediatric population.

All the collars tested in our study presented a statistical difference with the unbraced ROM. Statistical differences were found among collars in the primary plane of each movement and in the horizontal plane during lateral bending. Miami Jr and Necloc were shown to be the most effective in the limitation of the primary and coupled movements.

A large number of studies have compared the efficiency of different types of cervical collars in adults using several methods including x-rays, fluoroscopy, goniometry, ultrasounds, or infrared. ${ }^{4-9}$ However, there is no study analyzing pediatric cervical collars' efficiency on both primary and coupled movements. Studies conducted on adults, ${ }^{9}$ using radiographs in the neutral and maximal positions, showed similar results for Necloc and Miami Jr. In a study of Zhang et $a 1,{ }^{8}$ where the Vicon system was used, the results showed very good motion restriction by Miami Jr compared with three other orthoses used for adults.

The restriction of the neck's ROM, when collars are used on asymptomatic children, was described in our study. This could be seen as a limitation of this study when the results are applied on symptomatic subjects.

The normalized ROM, during a single-plane movement, were evaluated in the braced and unbraced conditions (Fig. 3). To our knowledge, this study is the first to evaluate the percentage of ROM (primary and coupled) by normalizing the results to the entire ROM in the 3 planes. The strategy of distribution between the primary and coupled planes, during a movement, differed between the braced and unbraced conditions.

When the flexion-extension of the neck was performed, the movement in the sagittal plane was less important in the braced condition, whereas, the movements in the frontal and horizontal planes were greater.

During left and right lateral bending, the movement was performed to a greater extent in the secondary planes (sagittal and horizontal) in the unbraced condition. Miami Jr and the Hard Collar showed a larger contribution in the frontal plane, with a reduced contribution of the horizontal plane. Necloc and Philadelphia reduced the movement without any change of the normalized ROM relatively to the unbraced condition.

During axial rotation, a similar panel to the flexionextension was present. All collars showed a statistical reduction of the normalized ROM in the horizontal plane and an increase in the secondary planes relatively to the unbraced condition.

These results showed that during the performance of flexion-extension and left-right axial rotation, the primary plane was the most used in the unbraced condition (around $80 \%$ ). In collar conditions, subjects performed a greater percentage of the movement in the secondary planes in response to the limitation of movement in the primary plane, caused by the collar.

However, a different strategy seemed to be used when left-right lateral bending was performed: the limitation caused by the collars increased the normalized ROM in the frontal plane (primary plane) and decreased it in the secondary planes, especially in the case of Miami $\mathrm{Jr}$ and the Hard Collar.

It would be pertinent to mention that as the anatomy and motion patterns of children over 8 years of age approach those of adults, the 6 to 12 age group is not helpful in extrapolating to small children but can only be interpreted for this age group.

A larger sample size could help us to be more decisive in the choice of the collar; however, the complexity of the coupled angles and the limited reproducibility of the children's positioning and movement performance still have to be taken into consideration.

In summary, Necloc and Miami Jr seem to be more efficient in limitation of primary and coupled movements. During lateral bending of the neck, in the unbraced condition, a large part of the movement was performed in the coupled planes. The strategy of distribution of the primary and coupled angles, during a single-plane movement, was often different between the unbraced and braced conditions but also within braced conditions. This finding emphasizes the impact of the choice of collars on the basis of the pathology and the need to limit neck movement in a specific plane.

\section{ACKNOWLEDGMENT}

The authors would like to thank Unicorn Lebanon for their financial support in this study. 


\section{REFERENCES}

1. Johnson RM, Hart DL, Simmons EF, et al. Cervical orthoses. A study comparing their effectiveness in restricting cervical motion in normal subjects. J Bone Joint Surg Am. 1977;59:332-339.

2. Richter D, Latta LL, Milne EL, et al. The stabilizing effects of different orthoses in the intact and unstable upper cervical spine: a cadaver study. J Trauma. 2001;50:848-854.

3. Sandler AJ, Dvorak J, Humke T, et al. The effectiveness of various cervical orthoses. An in vivo comparison of the mechanical stability provided by several widely used models. Spine (Phila Pa 1976). 1996;21:1624-1629.

4. Gavin TM, Carandang G, Havey R, et al. Biomechanical analysis of cervical orthoses in flexion and extension: a comparison of cervical collars and cervical thoracic orthoses. J Rehabil Res Dev. 2003;40: 527-537.

5. Hostler D, Colburn D, Seitz SR. A comparison of three cervical immobilization devices. Prehosp Emerg Care. 2009;13:256-260.

6. Quinlan JF, Mullett H, Stapleton R, et al. The use of the Zebris motion analysis system for measuring cervical spine movements in vivo. Proc Inst Mech Eng H. 2006;220:889-896.

7. Rosen PB, McSwain NE Jr, Arata M, et al. Comparison of two new immobilization collars. Ann Emerg Med. 1992;21:1189-1195.

8. Zhang S, Wortley M, Clowers K, et al. Evaluation of efficacy and 3D kinematic characteristics of cervical orthoses. Clin Biomech (Bristol, Avon). 2005;20:264-269.

9. Askins V, Eismont FJ. Efficacy of five cervical orthoses in restricting cervical motion. A comparison study. Spine (Phila Pa 1976). 1997; 22:1193-1198.

10. Dvir Z, Prushansky T, Peretz C. Maximal versus feigned active cervical motion in healthy patients: the coefficient of variation as an indicator for sincerity of effort. Spine (Phila Pa 1976). 2001;26: 1680-1688.

11. Reynolds J, Marsh D, Koller H, et al. Cervical range of movement in relation to neck dimension. Eur Spine J. 2009;18:863-868.

12. Malmstrom EM, Karlberg M, Fransson PA, et al. Primary and coupled cervical movements: the effect of age, gender, and body mass index. A 3-dimensional movement analysis of a population without symptoms of neck disorders. Spine (Phila Pa 1976). 2006; 31:E44-E50.

13. Demaille-Wlodyka S, Chiquet C, Lavaste JF, et al. Cervical range of motion and cephalic kinesthesis: ultrasonographic analysis by age and sex. Spine (Phila Pa 1976). 2007;32:E254-E261.
14. Youdas JW, Garrett TR, Suman VJ, et al. Normal range of motion of the cervical spine: an initial goniometric study. Phys Ther. 1992;72:770-780.

15. Ferrario VF, Sforza C, Serrao G, et al. Active range of motion of the head and cervical spine: a three-dimensional investigation in healthy young adults. J Orthop Res. 2002;20:122-129.

16. Sforza C, Grassi G, Fragnito N, et al. Three-dimensional analysis of active head and cervical spine range of motion: effect of age in healthy male subjects. Clin Biomech (Bristol, Avon). 2002;17: 611-614.

17. Arbogast KB, Gholve PA, Friedman JE, et al. Normal cervical spine range of motion in children 3-12 years old. Spine (Phila Pa 1976). 2007;32:E309-E315.

18. Lynch-Caris T, Majeske KD, Brelin-Fornari J, et al. Establishing reference values for cervical spine range of motion in pre-pubescent children. J Biomech. 2008;41:2714-2719.

19. Seacrist T, Saffioti J, Balasubramanian S, et al. Passive cervical spine flexion: the effect of age and gender. Clin Biomech (Bristol, Avon). 2012;27:326-333.

20. Roozmon P, Gracovetsky SA, Gouw GJ, et al. Examining motion in the cervical spine. II: Characterization of coupled joint motion using an opto-electronic device to track skin markers. J Biomed Eng. 1993;15:13-22.

21. Feipel V, Rondelet B, Le Pallec J, et al. Normal global motion of the cervical spine: an electrogoniometric study. Clin Biomech (Bristol, Avon). 1999;14:462-470.

22. Trott PH, Pearcy MJ, Ruston SA, et al. Three-dimensional analysis of active cervical motion: the effect of age and gender. Clin Biomech (Bristol, Avon). 1996;11:201-206.

23. Davis RB, Ounpuu S, Tyburski D, et al. A gait analysis data collection and reduction technique. Hum Mov Sci. 1991;10: 575-587.

24. Bland JM, Altman DG. Statistical methods for assessing agreement between two methods of clinical measurement. Lancet. 1986;1: 307-310.

25. Greaves LL, Van Toen C, Melnyk A, et al. Pediatric and adult three-dimensional cervical spine kinematics: effect of age and sex through overall motion. Spine (Phila Pa 1976). 2009;34:1650-1657.

26. Ishii T, Mukai Y, Hosono N, et al. Kinematics of the cervical spine in lateral bending: in vivo three-dimensional analysis. Spine (Phila Pa 1976). 2006;31:155-160.

27. Ishii T, Mukai Y, Hosono N, et al. Kinematics of the upper cervical spine in rotation: in vivo three-dimensional analysis. Spine (Phila Pa 1976). 2004;29:E139-E144. 